\title{
Effects of induction of anaesthesia on microvascular reactivity monitored by near-infrared spectroscopy
}

\author{
Vandenbulcke L, De Hert S, Moerman A \\ Ghent University Hospital, Dept of Anaesthesiology, Ghent, Belgium
}

\section{Background}

- Alterations in microvascular perfusion are associated with impaired tissue oxygenation and organ dysfunction.

- Microcirculation can be assessed by measurement of the postocclusive reactive hyperaemia (PORH) response.

- PORH is a reproducible transient increase in blood flow after release of an arterial occlusion.

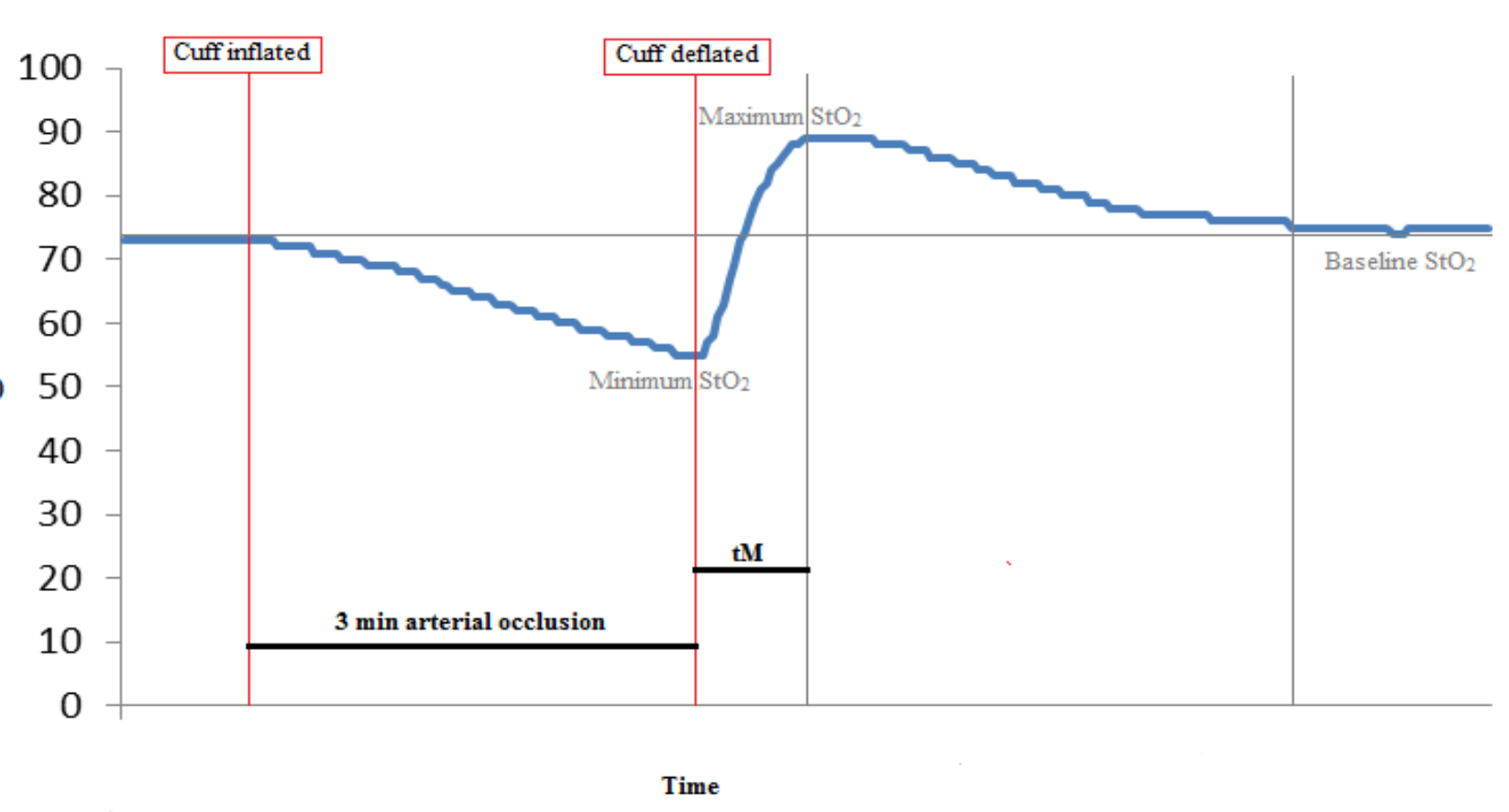

\section{Hypothesis}

We hypothesized that induction of anaesthesia would impair microvascular reactivity.

\section{Methods}

- 35 adult patients scheduled for elective coronary artery bypass grafting surgery

- PORH was measured with the use of near-infrared spectroscopy (NIRS, Foresight) before and 30 minutes after anaesthesia induction.

- Data were compared using the paired samples t-test or the Wilcoxon signed rank test.

\section{$\underline{\text { Results }}$}

- Tissue oxygen saturation was higher after induction of anaesthesia (median [min-max]: $73 \%$ [55-94] vs $70 \%$ [54-78], $\mathrm{p}=0.015)$.

- Oxygen consumption decreased after induction, appreciable by the higher minimum tissue oxygen saturation $(p<0.001)$ and the slower oxygen consumption rate $(\mathrm{p}<0.001)$.
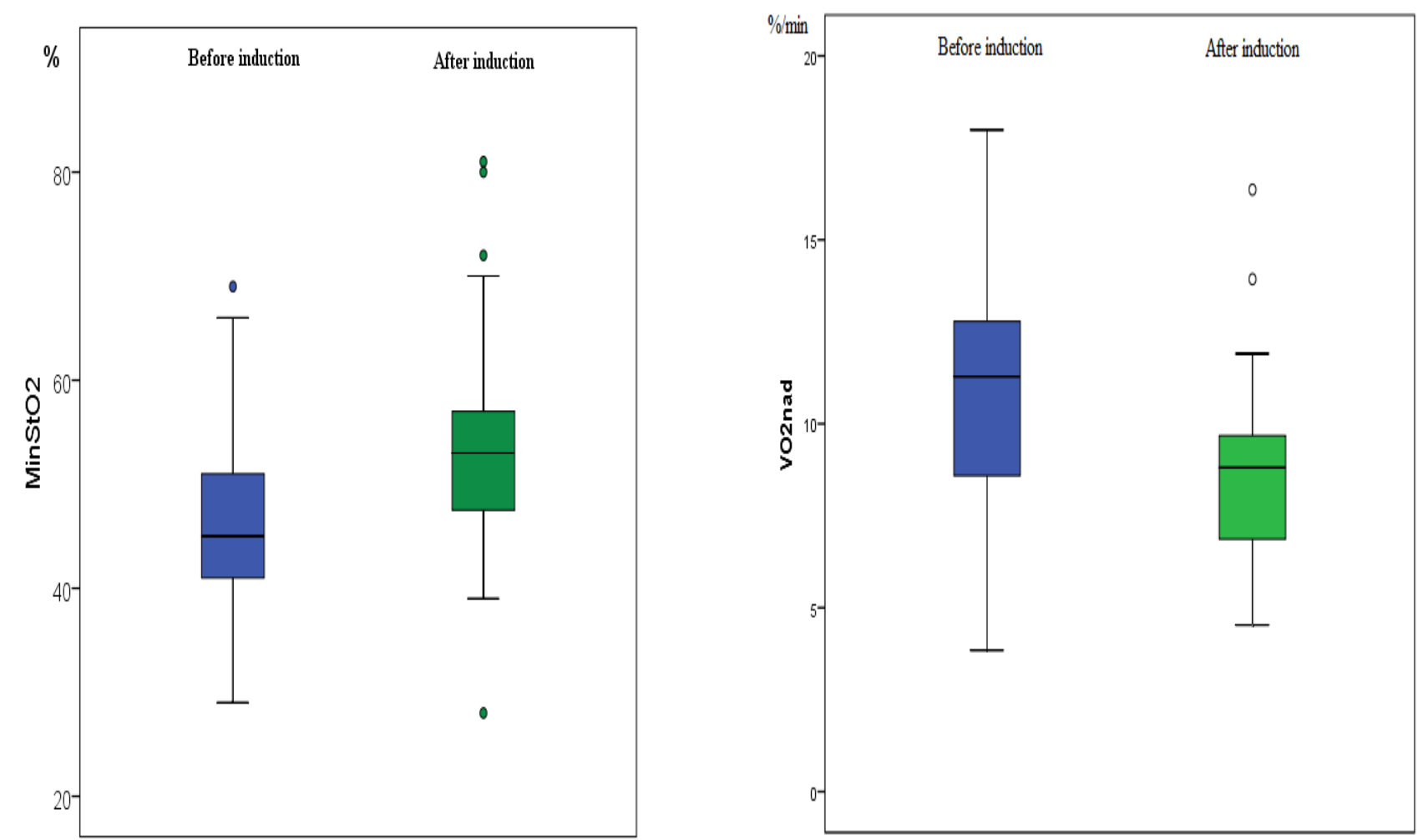

- After induction of anaesthesia, recovery times were longer $(p=0.004)$ and the rate of recovery was slower $(\mathrm{p}<0.001)$.
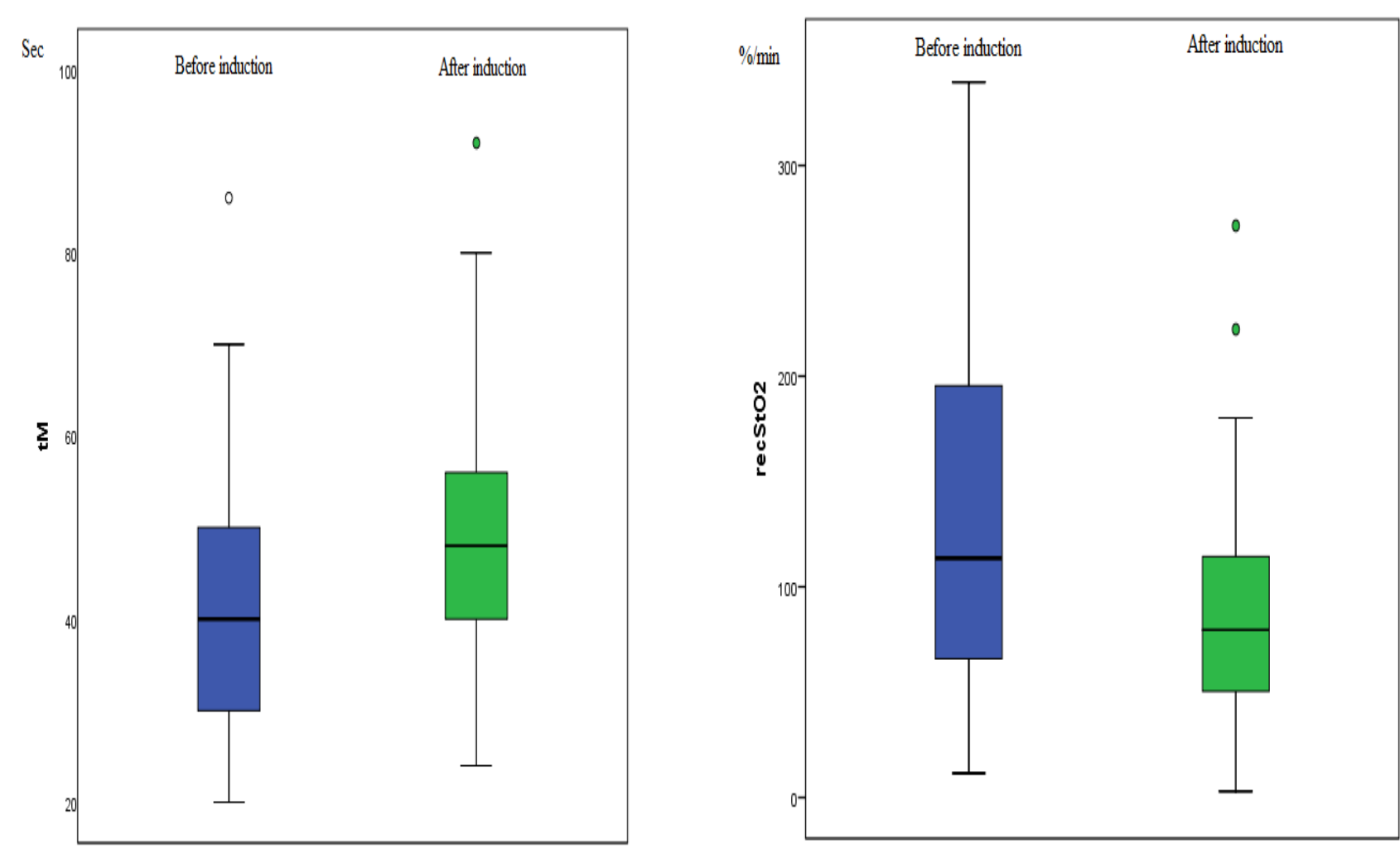

\section{Conclusion}

The longer recovery times and slower rates of recovery indicate impaired microvascular reactivity after induction of anaesthesia. 